\title{
Histomorphological study of corneal dystrophies
}

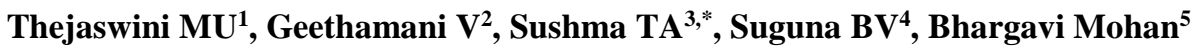 \\ ${ }^{1,3}$ Associate Professor, ${ }^{2}$ Professor \& HOD, ${ }^{4}$ Professor, ${ }^{5}$ Assistant Professor, Dept. of Pathology
}

*Corresponding Author:

Email: sushmapath@gmail.com

\begin{abstract}
Introduction: The term Corneal Dystrophy refers to a rare heterogeneous group of genetically determined, bilateral, symmetric, primary diseases, restricted to cornea and not associated with previous ocular inflammation. The dystrophies are classified based on the layer of cornea involved, into superficial, stromal and posterior dystrophies, each of which is genetically determined. Though the clinical features are often characteristic, definitive diagnosis is possible only after histological examination. The management protocols and visual prognosis vary with the underlying conditions. The incidence of the subtypes varies with the geographic locations. Objective: To study the prevalence of various subtypes of Corneal Dystrophies in corneal button specimens obtained after penetrating keratoplasty in a referral ophthalmic institute and to correlate with the patient's age and sex.

Materials and Methods: Corneal button specimens received in the ocular pathology laboratory over a period of five years were reviewed. Histopathological features of Corneal Dystrophies were studied and subcategorised. Cliniopathological analysis was made.

Results: Out of 660 corneal biopsies reviewed, 42 cases were of corneal dystrophy. The patients were between 2 and 71 years of age, with 14 males and 28 females. Macular dystrophy was the most common with 20 cases, followed by Congenital Hereditary Endothelial Dystrophy (CHED) and Fuch's endothelial dystrophy (FECD), with 7 cases each. Epithelial basement membrane dystrophy, Reis Buckler Dystrophy (RBCD), Granular dystrophy were the other types.

Conclusion: Histopathological sub categorisation of various types of Corneal Dystrophies not only helps in understanding the prevalence, but also in predicting the genetic link and prognosis of the disease category.
\end{abstract}

Keywords: Cornea, CHED, Dystrophy, Fuch, Macular.

Received: $29^{\text {th }}$ August, 2017

\section{Introduction}

Corneal Dystrophies are a rare group of primary diseases of cornea, which are generally bilateral and genetically determined. They are characterised by absence of any underlying predisposing factor such as inflammation or associated systemic illness. Corneal Dystrophies are classified into various subtypes based on the level of cornea affected, into Superficial (involvement of epithelial, basement membrane, Bowman's membrane and superficial stromal layers), Stromal (involvement of the stromal layer) and Posterior (involvement of Descemet's membrane and endothelium layer) dystrophies. The clinical management and visual prognosis following therapy vary with the type of dystrophy. The incidence of the different subtypes varies with the geographic locations, some being described only in specific ethnic groups. The present study was conducted to understand the prevalence of different subtypes of this rare disorder and to correlate with the patient profile.

\section{Material and Methods}

The study was conducted in a tertiary care ophthalmic institute in Southern India. All the corneal button specimens received in the ocular pathology laboratory over a period of five years were reviewed. Cases of Corneal Dystrophies were studied in detail and subcategorised, based on the level of cornea affected and nature of the deposits. Special stains such as Periodic Acid Schiff's stain, Alcian Blue stain were performed after taking sections from the paraffin blocks, to confirm the diagnosis, where necessary. The different categories were analyzed with the patient's age and sex. 


\section{Results}

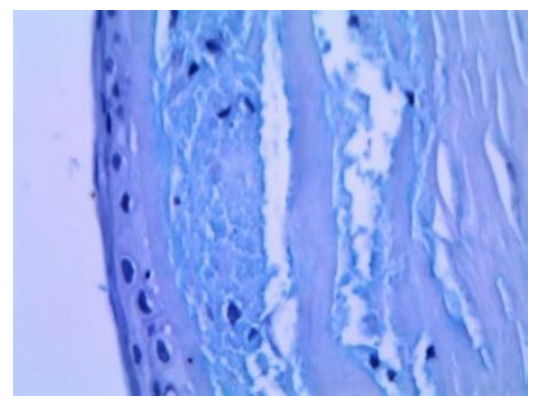

Fig. 1: Macular Corneal Dystrophy: Alcian Blue stain showing extracellular stromal deposition of glycosaminoglycans

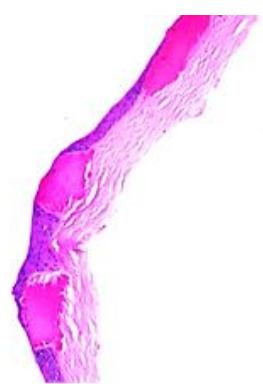

Fig. 2: Granular Corneal Dystrophy: Irregular deposits of eosinophilic material in the stroma

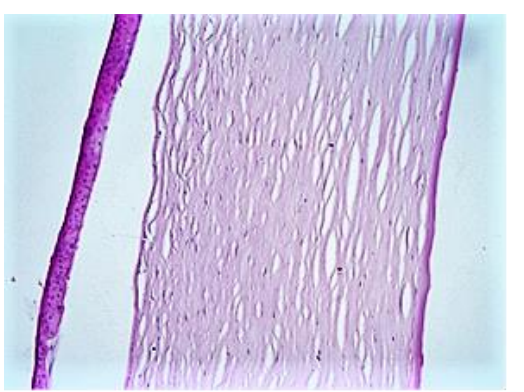

Fig. 3: Fuch's Corneal Dystrophy: A subepithelial bulla resulting from fluid accumulation beneath subepithelium

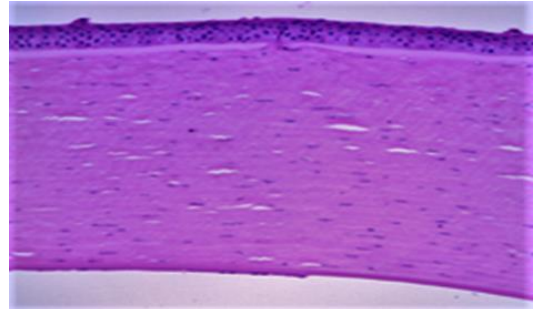

Fig. 4: Congenital Hereditary Endothelial Dystrophy: Light microscopy showing Corneal stromal edema

Out of 660 corneal biopsies submitted for histopathology study, 42 (6.4\%) cases belonged to Corneal Dystrophy. The patients were aged between 2 years and 71 years, with a mean of 45 years. Male: Female ratio was 1:2. Macular corneal dystrophy (MCD) was the commonest type, accounting for $47.6 \%$ (20) of the cases, followed by Congenital Hereditary Endothelial dystrophy (CHED) and Fuch's endothelial dystrophy (FECD), with $16.6 \%$ (7) each. Granular dystrophies accounted for $4.7 \%$ (2), while $9.5 \%$ (4) had overlapping features and specific categorisation was not possible. Epithelial basement membrane dystrophy and Reis Buckler Dystrophy were the least common type, with 1 case each (Table 1). Children and young adults in the age range of 2 years to 25 years constituted the bulk of CHED cases. The patients with Macular Corneal Dystrophy presented between 18 and 71 years age. Patients with Fuch's dystrophy were in the age group between 42 years and 65 years.

Table 1: Different types of Corneal dystrophies in the present study

\begin{tabular}{|l|c|}
\hline \multicolumn{1}{|c|}{ Type } & Number \\
\hline Macular Corneal Dystrophy & 20 \\
\hline $\begin{array}{l}\text { Congenital Hereditary Endothelial } \\
\text { Dystrophy. }\end{array}$ & 7 \\
\hline Fuch's Endothelial Dystrophy & 7 \\
\hline Granular Dystrophy & 2 \\
\hline Reis Buckler Dystrophy & 1 \\
\hline $\begin{array}{l}\text { Epithelial Basement Membrane } \\
\text { Dystrophy }\end{array}$ & 1 \\
\hline Unclassifiable & 4 \\
\hline Total & 42 \\
\hline
\end{tabular}

\section{Discussion}

The term Corneal Dystrophy refers to a group of inherited disorders of cornea which are bilateral, slowly progressive and bear no relation to the environmental or systemic factors. ${ }^{1}$ There are many exceptions to the said features; some of the entities are not hereditary, while others are unilateral. Incidence of each subtype varies in different geographic regions studied ${ }^{4-8}$. The worldwide prevalence of Corneal Dystrophies in corneal button specimens ranges from $1.3 \%$ to $4 \% .^{9,10}$ In our study, corneal dystrophies accounted for $6.4 \%$ of the total penetrating keratoplasty specimens. This slight increase may be due to higher incidence of consanguinity among the rural Southern Indian population.

The diagnosis of Corneal Dystrophy is made by clinical examination, direct and indirect ophthalmoscopy, confocal microscopy, confirmed by Histopathology, Transmission Electron microscopy and molecular genetic analysis. Histopathological examination of the corneal button specimens obtained after penetrating keratoplasty remains the gold 
standard tool in the diagnosis of Corneal Dystrophies.

Most of the dystrophies have Autosomal Dominant pattern of inheritance, start in the first decade of life, with stable or slowly progressive course. $^{5}$ The clinical presentations vary from photophobia, pain, blurring to progressive loss of vision. The most commonly used classification system is based on the anatomical level of cornea that is predominantly involved: Superficial, stromal and Posterior. Superficial dystrophies include pathology in the epithelial, basement membrane, Bowman's membrane and superficial stromal layers; in stromal dystrophies, only the corneal stroma is involved, while Posterior dystrophies exhibit Descemet membrane and corneal endothelium involvement. ${ }^{2}$

In our study, Macular Corneal Dystrophy was the most common type, which belongs to the group of corneal stromal dystrophies. The other types of stromal dystrophy are Granular, Lattice, Schnyder, Fleck, congenital stromal and Posterior amorphous corneal dystrophies. MCD is most prevalent in India, Saudi Arabia and also accounts for a majority of keratoplasties in Iceland. ${ }^{3}$ Similarly, in our study, MCD accounted for nearly $47 \%$ of cases of Corneal Dystrophies. In the present study, clinical presentation, age at keratoplasty was wide, ranged from 18 years to 70 years. Most cases of MCD are inherited in Autosomal recessive fashion, linked to mutation in CHST6 gene; present with progressive, severe visual impairment between 10 and 30 years of age. Reduction of corneal sensitivity and photophobia may be present. Course is slowly progressive. Light Microscopy shows Glycosaminoglycans (GAGs), which accumulate both intracellularly and extracellularly in the corneal stroma, also in Descemet membrane and corneal endothelium. The deposits stain positively with histochemical stains such as PAS, Alcian blue or Hale colloidal iron. Guttae are commonly present on Descemet membrane. In the present study, Alcian Blue positive deposition was seen predominantly in the corneal stroma (Fig. 1).The Descemet membrane and the endothelium were largely unremarkable.

Granular Corneal Dystrophy (GCD), which is a type of stromal dystrophy with Autosomal Dominant inheritance, was the third commonest in the present study, second being the posterior dystrophies such as FECD and CHED. Both the patients of GCD were males. GCD is an autosomal dominant disease, patients develop sharply demarcated bread crumb like opacities in the central cornea in the first decade of life. Visual acuity is not impaired till late stages of the disease. Histologically, abnormal eosinophilic granules are deposited in the corneal stroma (Fig. 2), which stains brilliant red with Masson Trichrome. They consist predominantly of transforming growth factor $\beta$ induced protein (TGF $\beta I$ ). ${ }^{3}$ The opacities may recur in the graft within a year of penetrating keratoplasty. ${ }^{3}$

The other forms of stromal dystrophy such as Lattice type, Schnyder, Fleck, Congenital stromal and posterior amorphous were not encountered in the present study.

FECD and CHED are the second common type of dystrophies in the present study. Both the types belong to the posterior corneal dystrophies; other posterior dystrophies are Posterior Polymorphous Corneal Dystrophy (PPCD), X linked endothelial corneal dystrophy (XECD). All the posterior dystrophies are characterised by abnormalities of corneal endothelium and Descemet membrane. In the present study, only CHED and FECD were seen, which accounted for $16.6 \%$ of cases each.

FECD is the most prevalent type of corneal dystrophy in the USA and other developed countries, considered as an Autosomal Dominant, slowly progressive corneal disorder of aging. FECD is more common and more severe in women (3-4:1), ${ }^{3}$ while in our study, the ratio was 1.3:1 and patients were aged between 42-65 years. The classical findings in FECD are corneal edema, golden brown guttae on slit lamp examination and reduced visual acuity. Initially, stromal edema is the only change that can be observed. Fluid accumulates in between the epithelium and subepithelium (Fig. 3), which may burst, causing epithelial erosions. Histopathological features of FECD are corneal guttae (excrescences) on Descemet's membrane, in the central region of cornea. Corneal erosions and cataracts are common. FECD is considered as a complex inherited disorder, with the exact cause not yet known. Reasons for increased incidence in women are yet to be determined. Most cases require penetrating keratoplasty or advanced procedures for repairing the posterior surface of cornea.

Congenital Hereditary Endothelial Dystrophy (CHED) presents as bilateral diffuse ground glass opacities with thickened corneas from birth or infancy. Two subtypes of CHED are described, based on the inheritance pattern and clinical presentation. CHED1 (Autosomal Dominant) presents in the first two years of life, with photophobia. CHED2 (Autosomal recessive) manifests in young children, with nystagmus. Histologically, both the types are similar, with stromal edema, enlargement of collagen fibres, scant/ degenerated endothelium 
(Fig. 4). The corneal thickness is markedly increased. CHED1 is slowly progressive while CHED2 is stationary. Penetrating keratoplasty is essential when the opacities are severe.

Superficial dystrophies include Meesman Dystrophy (MECD), Reiss-Buckler dystrophy (RBCD), Thiel-Behnke dystrophy (TBCD), Gelatinous Drop like corneal dystrophy (GDCD) and many others. All these entities are rare, seen only in specific populations and ethnic descents. However, in the present study, one case each of Epithelial Basement Membrane Dystrophy and RBCD were seen. RBCD is an Autosomal Dominant disease, associated with mutation in TGFB1 gene, present with pain, discomfort and progressive loss of vision. In RBCD, patients classically present with symmetrical reticular opacities in the third decade of life. As the superficial corneal stroma contains deposition of mutated transforming factor $\beta$ induced protein, it appears similar to Granular corneal dystrophy. Clinical correlation and genetic studies aid in the diagnosis of this subtype. Superficial keratectomy or lamellar keratoplasty are essential to reduce the symptoms.

In 4 cases, exact sub categorisation was not possible, as they had features of degenerative changes of multiple layers, with fibrosis and increased vascularity. These patients had presented in later stages of the disease, after many years of impaired vision. Such cases are difficult to subcategorise.

\section{Conclusion}

Corneal dystrophies are an uncommon group of disorders with clinical presentation and prognosis specific to the histopathological subtype involved. In the Indian subcontinent, Macular Corneal dystrophy is the most common type encountered. With the advancement in the field of diagnostic ophthalmoscopy, a clinical diagnosis of corneal dystrophy may be made easily. Appearance of cornea on slit lamp microscopy, confocal microscopy further aid in the disease categorisation. However, precise diagnosis can be established by histopathological examination of the excised corneal tissue by light microscopy, followed by Electron microscopy and molecular genetics. In a resource limited country, histopathological examination with special stains can identify with fair accuracy, already genetically established clinical entities and prove valuable in providing genetic counselling, therapeutic options and prognostication.

\section{Acknowledgements}

The authors wish to express their gratitude and sincere thanks to the staff and Management of Laboratory at Narayana Nethralaya, Electronic City, Bangalore for their support and cooperation.

\section{References}

1. American Academy of Ophthalmology. External diseases and cornea. In: Sutphin JE, ed. Basic and Clinical Sciences Course 2007-2008. San Francisco, CA: American Academy of Ophthalmology;2007:305-29.

2. Weiss JS, Moller HU, Lisch W, Kinoshita S, Aldave AJ, Belin MW, et al "The IC3D Classification of the Corneal Dystrophies" Cornea (2008) 27 Suppl. 2, 81-3.

3. Klintworth GK "Corneal Dystrophies" Orphanet J Rare Dis (2009) 4-7.

4. Pandrowala H, Bansal A, Vemuganti GK, Rao GN "Frequency, distribution, and outcome of keratoplasty for corneal dystrophies at a tertiary eye care center in South India" Cornea(2004) 23(6), 541-6.

5. Abreu EB, Novaes GA, Fernandes BF, Odashiro PRP, Odashiro AN, Parizotto IOL, Et.al "Corneal stromal dystrophies: a clinicopathological study" Arq Bras Oftalmol (2012) 75(6), 390-3.

6. Faran MF, Tabbara KF "Corneal dystrophies among patients undergoing keratoplasty in Saudi Arabia" Cornea (1991) 10(1),13-6.

7. Lang GK, Naumann GO "The frequency of corneal dystrophies requiring keratoplasty in Europe and the USA" Cornea (1987) 693,209-11.

8. Al-Yousuf N, Mavrikaksi E, Daya SM "Penetrating keratoplasty: indications over a 10 year period" Br J Ophthalmol (2004) 88(8), 9981001.

9. Santos LN, Fernandes BF, de Moura LR, Cheema DP, Maloney S, Logan P, et al "Histopathologic study of corneal stromal dystrophies: a 10-year experience" Cornea (2007) 26(9), 1027-31.

10. Cosar CB, Sridhar MS, Cohen EJ, Held EL, Alvim P deT, Rapuanao CJ, et al "Indications for penetrating keratoplasty and associated procedures, 1996-2000" Cornea (2002) 21(2), $148-51$. 